\title{
Boundary conditions for surface reactions in lattice Boltzmann simulations
}

\author{
J. J. J. Gillissen* and N. Looije \\ Department of Chemical Engineering, Delft University of Technology, Julianalaan 136, 2628 BL Delft, the Netherlands
}

(Received 29 November 2013; published 18 June 2014)

\begin{abstract}
A surface reaction boundary condition in multicomponent lattice Boltzmann simulations is developed. The method is applied to a test case with nonlinear reaction rates and nonlinear density profiles. The results are compared to the corresponding analytical solution, which shows that the error of the method scales with the square of the lattice spacing.
\end{abstract}

DOI: 10.1103/PhysRevE.89.063307

PACS number(s): 47.11.-j, 82.20.Wt

\section{INTRODUCTION}

The lattice Boltzmann (LB) method is a computational fluid dynamics method, which is particularly suitable for multiphase flows and complexly shaped boundaries; see, e.g., Refs. [1-5]. The LB method is based on a discretization of the Boltzmann equation, governing the single-particle distribution function of position and momentum.

In this paper the LB method is applied to chemical surface reactions. The focus is on the formulation of the surface reaction boundary conditions within the LB framework. LB boundary conditions for surface reactions have previously been addressed in the literature; see, e.g., Refs. [6-19]. Details of these studies are explained in Sec. IV. To the best of the authors' knowledge all previously proposed implementations of surface reaction boundary conditions in LB simulations require iterative, root-finding methods to deal with nonlinear reaction rates, i.e., reaction rates that are nonlinear functions of the component densities at the wall; see, e.g., Refs. [12-14,17]. The goal of the present work is to develop an explicit implementation of the surface reaction boundary condition within the multicomponent LB framework. The advancement of the current scheme over previously proposed schemes is that it consists of a single, fully explicit expression and that it requires no iterations to deal with nonlinear reaction rates. In this respect it is noted that Huber et al. also implement nonlinear reaction rates explicitly [19]. Their method replaces the boundary condition by a volumetric source term, which is different from enforcing the boundary condition directly on the surface, which is the approach adopted in the present work.

The remainder of the paper is organized as follows. Section II introduces the variables that define multicomponent surface reactions. Section III presents the LB method, and Sec. IV presents the surface reaction boundary condition. The main result is presented by Eq. (14). Then Sec. V presents a test case and compares the outcome of the LB simulations to the corresponding analytical solution. Conclusions are summarized in Sec. VI.

The main conclusion is that the error of the method scales with the square of the grid spacing; i.e., the method is second-order accurate. Although the test case is in one spatial dimension, the method can be directly applied to complexly shaped boundaries in more dimensions, as long as the shapes are approximated by staircases, whose surfaces lie halfway between the lattice sites, which is the conventional approxi-

*jurriaangillissen@gmail.com mation used in LB simulations. It is known, however, that the staircase approximation reduces the order of the accuracy of the LB method from two to one; see, e.g., Ref. [20]. To maintain second-order accuracy for complexly shaped boundaries, the present approach could be combined with an interpolation treatment of curved boundaries; see, e.g., Ref. [21].

\section{PROBLEM FORMULATION}

An ideal fluid mixture of $N$ components is considered, where $m_{\sigma}$ is the molecular mass of component $\sigma=$ $\{1,2, \ldots, N\}$. The fluid mixture reacts on a wall. The reaction can also involve wall molecules of mass $m_{w}$. The mass balance of the reaction is

$$
\sum_{\sigma} \xi_{\sigma} m_{\sigma}+\xi_{w} m_{w}=0
$$

where $\xi_{\sigma}$ and $\xi_{w}$ are the stoichiometrics of component $\sigma$ and of the wall component, respectively, and the summation $\sum_{\sigma}$ runs from $\sigma=1$ to $N$. Depending on whether component $\sigma$ is produced or consumed in the reaction, $\xi_{\sigma}>0$ or $\xi_{\sigma}<0$, respectively. Due to the surface reaction there is a wall normal mass flux for each component $\Phi_{\sigma}$. It is assumed that the wall is located at $x=0$ and the fluid resides at $x>0$. A positive or a negative $\Phi_{\sigma}$ corresponds to production or consumption of component $\sigma$, respectively. Since $\Phi_{\sigma}$ is proportional to $\xi_{\sigma} m_{\sigma}$, the mass balance [Eq. (1a)] can also be written as

$$
\sum_{\sigma} \Phi_{\sigma}+\Phi_{w}=0
$$

where $\Phi_{w}$ is the wall normal mass flux of the wall component. The reaction rate is defined as the production of component one $\Phi_{1}$, which is a generic function of the various component densities $\rho_{\sigma}$ at the wall:

$$
\Phi_{1}=R\left(\rho_{\sigma}\right) .
$$

The wall normal mass fluxes of the other components are given by

$$
\Phi_{\sigma}=\Phi_{1} \frac{\xi_{\sigma} m_{\sigma}}{\xi_{1} m_{1}}
$$

\section{LATTICE BOLTZMANN MODEL}

In this work an implementation is developed for surface reaction boundary conditions in multicomponent LB simulations. For the sake of generality these boundary conditions are formulated in an arbitrary number $D$ of spatial dimensions. 
The LB method solves the single particle distribution function of position and momentum. In the standard LB method the $D$-dimensional position space is decomposed into lattice sites, which are separated in each dimension by the same distance $\Delta x$, and the $D$-dimensional velocity space is discretized into a set of $Q$ discrete velocities $\boldsymbol{v}_{\alpha}$ that per time step $\Delta t$ correspond to translations between neighboring lattice sites. For the sake of completeness Table I in Appendix A lists three commonly used velocity sets in $D=1,2$, and 3 dimensions, consisting of $Q=3,9$, and 19 velocities, which are referred to as D1Q3, D2Q9, and D3Q19. The distribution function for fluid component $\sigma$ and for velocity $\boldsymbol{v}_{\alpha}$ is denoted $f_{\sigma, \alpha}$. The $f_{\sigma, \alpha}$ are also referred to as LB particles. The dimension of $f_{\sigma, \alpha}$ is mass per volume.

The component density $\rho_{\sigma}$ and the component velocity $\boldsymbol{u}_{\sigma}$ are related to the distribution function $f_{\sigma, \alpha}$ by $\rho_{\sigma}=\sum_{\alpha} f_{\sigma, \alpha}$ and $\boldsymbol{u}_{\sigma}=\sum_{\alpha} f_{\sigma, \alpha} \boldsymbol{v}_{\alpha} / \rho_{\sigma}$, respectively, where the summation $\sum_{\alpha}$ runs from $\alpha=1$ to $Q$. The total mass density and the mass averaged velocity are given by $\rho=\sum_{\sigma} \rho_{\sigma}$ and $\boldsymbol{u}=$ $\sum_{\sigma} \rho_{\sigma} \boldsymbol{u}_{\sigma} / \rho$, respectively.

The function $f_{\sigma, \alpha}$ is defined on each lattice site and evolves in time according to the LB equation [4]:

$$
\frac{f_{\sigma, \alpha}\left(\boldsymbol{x}+\Delta t \boldsymbol{v}_{\alpha}, t+\Delta t\right)-f_{\sigma, \alpha}(\boldsymbol{x}, t)}{\Delta t}=\frac{f_{\sigma, \alpha}^{e q}(\boldsymbol{x}, t)-f_{\sigma, \alpha}(\boldsymbol{x}, t)}{\tau_{\sigma}},
$$

where $\tau_{\sigma}$ is the relaxation time of component $\sigma$ and $f_{\sigma, \alpha}^{e q}$ is the equilibrium distribution function, which is usually a truncated Mach number expansion of the Maxwell Boltzmann distribution function [4]. Here a modified version of $f_{\sigma, \alpha}^{e q}$ is used that allows the component sound speed $c_{\sigma}$ to be varied, which is normally fixed at $c_{0}=\Delta x / \sqrt{3} \Delta t$ [22]:

$$
f_{\sigma, \alpha}^{e q}=\rho_{\sigma}\left\{\gamma_{\alpha}+w_{\alpha}\left[\frac{\boldsymbol{v}_{\alpha} \cdot \boldsymbol{u}^{e q}}{c_{0}^{2}}+\frac{1}{2} \frac{\left[\boldsymbol{v}_{\alpha} \cdot \boldsymbol{u}^{e q}\right]^{2}}{c_{0}^{4}}-\frac{1}{2} \frac{\boldsymbol{u}^{e q 2}}{c_{0}^{2}}\right]\right\},
$$

where

$$
\gamma_{\alpha}= \begin{cases}w_{\alpha}+\left[1-w_{\alpha}\right]\left\{1-\left[\frac{c_{\sigma}}{c_{0}}\right]^{2}\right\} & \text { if } \boldsymbol{v}_{\alpha}=\mathbf{0} \\ w_{\alpha}\left[\frac{c_{\sigma}}{c_{0}}\right]^{2} & \text { if } \boldsymbol{v}_{\alpha} \neq \mathbf{0} .\end{cases}
$$

Other forms that allow a varying $c_{\sigma}$ are also possible; see, e.g., Ref. [23]. In Eqs. (4b)-(4c) $w_{\alpha}$ are the LB distribution weights. The values of these weights for D1Q3, D2Q9, and D3Q19 are listed in Table I. One time step of the LB equation [Eq. (4a)] involves two steps. In the streaming step, the LB particles move between neighboring lattice sites, carrying mass $f_{\sigma, \alpha}$ and momentum $f_{\sigma, \alpha} \boldsymbol{v}_{\alpha}$ of component $\sigma$ in direction $\boldsymbol{v}_{\alpha}$. The relaxation step "pushes" $f_{\sigma, \alpha}$ towards $f_{\sigma, \alpha}^{e q}$, such that the various components equilibrate towards a common velocity $\boldsymbol{u}^{e q}$. In order for the method to satisfy overall momentum conservation, this velocity must be equal to

$$
\boldsymbol{u}^{e q}=\frac{\sum_{\sigma} \rho_{\sigma} \boldsymbol{u}_{\sigma} \tau_{\sigma}^{-1}}{\sum_{\sigma} \rho_{\sigma} \tau_{\sigma}^{-1}} .
$$

It can be shown that the LB method [Eq. (4)] recovers the correct macroscopic component mass balance:

$$
\frac{\partial \rho_{\sigma}}{\partial t}+\nabla \cdot\left(\boldsymbol{u} \rho_{\sigma}\right)=\nabla \cdot D_{\sigma} \nabla \rho_{\sigma}
$$

where, provided that there is no pressure gradient and that the $\tau_{\sigma}$ are all equal, the component mass diffusivity $D_{\sigma}$ is given by [24]

$$
D_{\sigma}=c_{\sigma}^{2}\left[\tau_{\sigma}-\frac{\Delta t}{2}\right]
$$

\section{SURFACE REACTION BOUNDARY CONDITION}

LB boundary conditions are usually referred to as bounceback methods, since one can imagine LB particles hitting a wall and bouncing back into the fluid domain. These methods can be divided into on-grid and halfway bounce-back methods, depending on whether the wall is located on the lattice sites or halfway between them. Different types of boundary conditions can be realized, depending on the values for the outgoing particles as functions of the incoming particles.

Surface reaction boundary conditions have been developed in the literature following roughly three approaches. The first approach is based on the on-grid bounce-back method by He et al. [25], who use that at the wall the nonequilibrium part of the distribution function: $f_{\sigma, \alpha}-f_{\sigma, \alpha}^{e q}$ is proportional to the dot product of the concentration gradient $\nabla \rho_{\sigma}$ and the microscopic velocity $\boldsymbol{v}_{\alpha}$; see, e.g., Refs. $[7,8,12,18]$. The second approach is based on the so-called diffusive, halfway, bounce-back method by Ansumali and Karlin [26], who assume Maxwellian outgoing particles; see, e.g., Refs. [13,16]. The third approach to LB surface reactions is based on a principle proposed by Ladd [20] of adding a source term to the halfway bounce-back method to realize a wall normal mass flux; see, e.g., Refs. [11,14,15,27].

A wall is assumed at $x=0$ and a fluid at $x>0$ and an incoming particle $f_{\sigma, \alpha}^{-}$is emitted from a lattice site next to the wall at $x=\Delta x / 2$ with a wall normal velocity $v_{\alpha, x}<0$. Upon bouncing back from the wall into the fluid domain, its velocity is reversed from $\boldsymbol{v}_{\alpha}$ to $\boldsymbol{v}_{\bar{\alpha}}=-\boldsymbol{v}_{\alpha}$. Due to the surface reaction the particle mass changes during the bounce-back, which induces the component wall normal mass flux $\Phi_{\sigma}$. Using Ladd's principle the outgoing particle $f_{\sigma, \bar{\alpha}}^{+}$is written as the following function of the incoming particle $f_{\sigma, \alpha}^{-}$:

$$
f_{\sigma, \bar{\alpha}}^{+}=f_{\sigma, \alpha}^{-}+6 w_{\alpha}\left(\frac{\Delta t}{\Delta x}\right)^{2} \boldsymbol{v}_{\bar{\alpha}} \cdot \boldsymbol{n} \Phi_{\sigma},
$$

where $\boldsymbol{n}$ is the wall normal unit vector pointing into the fluid.

By combining Eqs. (2), (3), and (7) the following surface reaction bounce-back method is obtained:

$$
f_{\sigma, \bar{\alpha}}^{+}=f_{\sigma, \alpha}^{-}+6 w_{\alpha}\left(\frac{\Delta t}{\Delta x}\right)^{2} \boldsymbol{v}_{\bar{\alpha}} \cdot \boldsymbol{n} \frac{\xi_{\sigma} m_{\sigma}}{\xi_{1} m_{1}} R\left(\rho_{\sigma}(0)\right) .
$$

The reaction rate $R$ in Eq. (8) depends on the component densities at the wall $\rho_{\sigma}(0)$. Since the wall is located in between the lattice sites, the values of $\rho_{\sigma}(0)$ are not available during the course of a simulation. Therefore a first-order Taylor expansion is used,

$$
R\left(\rho_{\sigma}(0)\right)=R-\frac{\Delta x}{2}\left[\sum_{\sigma} \frac{\partial R}{\partial \rho_{\sigma}} \frac{\partial \rho_{\sigma}}{\partial x}\right],
$$

to express $R\left(\rho_{\sigma}(0)\right)$ in terms of known quantities at the nearest lattice site to the wall, which is at $x=\Delta x / 2$. All variables on the r.h.s. of Eq. (9) are evaluated at $x=\Delta x / 2$. This expansion 
is the main assumption of the present surface reaction bounceback method. The error that is introduced by this assumption is analyzed below, but first the derivation of the bounce-back method is completed. The wall normal mass flux is written as the sum of the advective and the diffusive flux:

$$
\Phi_{\sigma}=\rho_{\sigma} u-D_{\sigma} \frac{\partial \rho_{\sigma}}{\partial x},
$$

where $D_{\sigma}$ is the component mass diffusivity and $u$ is the mass averaged wall normal velocity:

$$
u=\frac{\sum_{\sigma} \Phi_{\sigma}}{\rho}=-\frac{\Phi_{w}}{\rho}=-\frac{\Phi_{1} \xi_{w} m_{w}}{\rho \xi_{1} m_{1}}
$$

where Eqs. (1b) and (3) have been used. Combining Eqs. (10) and (11) gives

$$
\frac{\partial \rho_{\sigma}}{\partial x}=-\frac{\Phi_{1}}{a D_{\sigma}}\left[\frac{\rho_{\sigma} \xi_{w} m_{w}}{\rho \xi_{1} m_{1}}+\frac{\xi_{\sigma} m_{\sigma}}{\xi_{1} m_{1}}\right] .
$$

Inserting Eq. (12) into Eq. (9) and solving for $\Phi_{1}=R\left(\rho_{\sigma}(0)\right)$ gives

$$
R\left(\rho_{\sigma}(0)\right)=\frac{R}{1-\frac{\Delta x}{2} \sum_{\sigma} \frac{1}{D_{\sigma}} \frac{\partial R}{\partial \rho_{\sigma}}\left[\frac{\rho_{\sigma} \xi_{w} m_{w}}{\rho \xi_{1} m_{1}}+\frac{\xi_{\sigma} m_{\sigma}}{\xi_{1} m_{1}}\right]} .
$$

By combining Eqs. (8) and (13), the following surface reaction bounce-back method is obtained:

$$
f_{\sigma, \bar{\alpha}}^{+}=f_{\sigma, \alpha}^{-}+\frac{6\left(\frac{\Delta t}{\Delta x}\right)^{2} w_{\alpha} \boldsymbol{v}_{\bar{\alpha}} \cdot \boldsymbol{n} \frac{\xi_{\sigma} m_{\sigma}}{\xi_{1} m_{1}} R}{1-\frac{\Delta x}{2} \sum_{\sigma^{\prime}} \frac{1}{D_{\sigma^{\prime}}} \frac{\partial R}{\partial \rho_{\sigma^{\prime}}}\left[\frac{\rho_{\sigma^{\prime}} \xi_{w} m_{w}}{\rho \xi_{1} m_{1}}+\frac{\xi_{\sigma^{\prime}} m_{\sigma^{\prime}}}{\xi_{1} m_{1}}\right]} .
$$

Equation (14) is the main result of the present paper. It is a surface reaction, bounce-back method within a multicomponent LB framework. In this equation, the reaction rate $R$ and its derivatives $\partial R / \partial \rho_{\sigma}$ as well as the component mass diffusivities $D_{\sigma}$ are allowed to depend on the various component densities, whose values are taken from the nearest lattice site to the wall.

The error of the present surface reaction bounce-back method [Eq. (14)] originates from the Taylor expansion [Eq. (9)], which involves first-order derivatives: $\partial R / \partial \rho_{\sigma}$ and $\partial \rho_{\sigma} / \partial x$. This expansion is needed to express the reaction rate at the wall in terms of quantities, which are defined on the lattice sites. When both $R\left(\rho_{\sigma}\right)$ and $\rho_{\sigma}(x)$ are linear functions, then this expansion is exact. In any other case, a relative error $\epsilon$ is introduced by this expansion, which can be estimated with the next term in the expansion [Eq. (9)] divided by $R$ :

$$
\epsilon=\left[\sum_{\sigma} \frac{\partial R}{\partial \rho_{\sigma}} \frac{\partial^{2} \rho_{\sigma}}{\partial x^{2}}+\sum_{\sigma \sigma^{\prime}} \frac{\partial^{2} R}{\partial \rho_{\sigma} \partial \rho_{\sigma^{\prime}}} \frac{\partial \rho_{\sigma}}{\partial x} \frac{\partial \rho_{\sigma^{\prime}}}{\partial x}\right] \frac{\Delta x^{2}}{8 R} .
$$

Equation (15) estimates the error in terms of the second-order derivatives: $\partial^{2} R / \partial \rho_{\sigma}^{2}$ and $\partial^{2} \rho_{\sigma} / \partial x^{2}$. These second derivatives are referred to as the "reaction curvature" and the "density curvature," respectively. Furthermore, the $\Delta x^{2}$ dependence in Eq. (15) suggests that the method is second-order accurate.

\section{TEST CASE}

To verify Eq. (15) a test case is designed in which both the reaction curvature $\partial^{2} R / \partial \rho_{\sigma}^{2}$ and the density curvature $\partial^{2} \rho_{\sigma} / \partial x^{2}$ can be varied. The test case consists of a steady, one-dimensional (1D) mixture of two ideal gases: $\mathrm{A}$ and
$\mathrm{B}$, bounded by two walls at $x=0$ and $x=L$. The volume averaged mass density $\bar{\rho}$ is given by

$$
\bar{\rho}=\frac{1}{L} \int_{0}^{L}\left[\rho_{A}(x)+\rho_{B}(x)\right] d x .
$$

The gases have molecular masses $m_{A}$ and $m_{B}$ and sound speeds $c_{A}=\sqrt{k_{B} T / m_{A}}$ and $c_{B}=\sqrt{k_{B} T / m_{B}}$, where $k_{B}$ is the Boltzmann constant and $T$ is the temperature. The component mass diffusivities $D_{\sigma}$ in the binary ideal gas mixture are given by

$$
D_{\sigma}=\frac{c_{\sigma}^{2}\left[m_{A}+m_{B}\right]}{\rho \Omega \sqrt{c_{A}^{2}+c_{B}^{2}}},
$$

where $\Omega$ is a molecular collision cross section; see, e.g., Ref. [28]. For the sake of completeness a derivation of Eq. (17) is provided in Appendix B. On $x=0$ component B is transformed into component $\mathrm{A}$ and on $x=L$ component $\mathrm{A}$ is transformed into component $\mathrm{B}$, by means of surface reactions. The corresponding reaction rates are $n$ th-order power functions of the reactant concentrations:

$$
R=k \rho_{B}^{n} \quad \text { for } x=0, \quad R=k \rho_{A}^{n} \quad \text { for } x=L,
$$

where $k$ is the reaction rate constant and $n$ is the reaction rate order. Both reactions at $x=0$ and $x=L$ have the following stoichiometric ratio:

$$
\frac{m_{A} \xi_{A}}{m_{B} \xi_{B}}=-1 .
$$

The analytical solution to the component mass density profiles and the reaction mass flux $\Phi_{A}$ are derived in Appendix C. The mass flux is plotted in Fig. 1 as a function of the independent parameters; i.e., the reaction rate constant $k$, the reaction rate order $n$, and the sound speed ratio $c_{B} / c_{A}$. For small $k$ the mass flux is proportional to $k$ and for large $k$ the mass flux saturates to a constant proportional to the logarithm of the sound speed ratio: $\Phi_{A} \sim \log \left(c_{B} / c_{A}\right)$. The densities are exponential functions of the position, unless both sound speeds are equal: $c_{A} / c_{B}=1$, in which case the density profiles are linear. The growth factor $\kappa$ of the exponential profiles is proportional to the reaction mass flux, and for $k \rightarrow \infty$ its nondimensional value equals $\kappa L=2 \log \left(c_{B} / c_{A}\right)$. The corresponding density profiles are illustrated in Fig. 2 for $c_{B} / c_{A}=3$.

The present test case allows varying the density curvature $\partial^{2} \rho_{\sigma} / \partial x^{2}$ and the reaction curvature $\partial^{2} R / \partial \rho_{\sigma}^{2}$, by varying the sound speed ratio $c_{A} / c_{B}$ and the reaction rate order $n$, respectively. This allows a systematic verification of the error [Eq. (15)] of the bounce-back method [Eq. (14)].

The test case is simulated using D1Q3; see Table I. In order for the LB component mass diffusivity [Eq. (6)] to recover the "real" diffusivity [Eq. (17)], both $\tau_{\sigma}$ are assumed to be the following function of the mass density:

$$
\tau_{\sigma}=\frac{\Delta t}{2}+\frac{m_{A}+m_{B}}{\rho \Omega \sqrt{c_{A}^{2}+c_{B}^{2}}} .
$$

It is noted that in addition to $D_{\sigma}$, the value of $\tau_{\sigma}$ also determines the kinematic viscosity. The present test case, however, concerns diffusive mass transport only; i.e., there is no advection and the viscosity of the system does not play a 

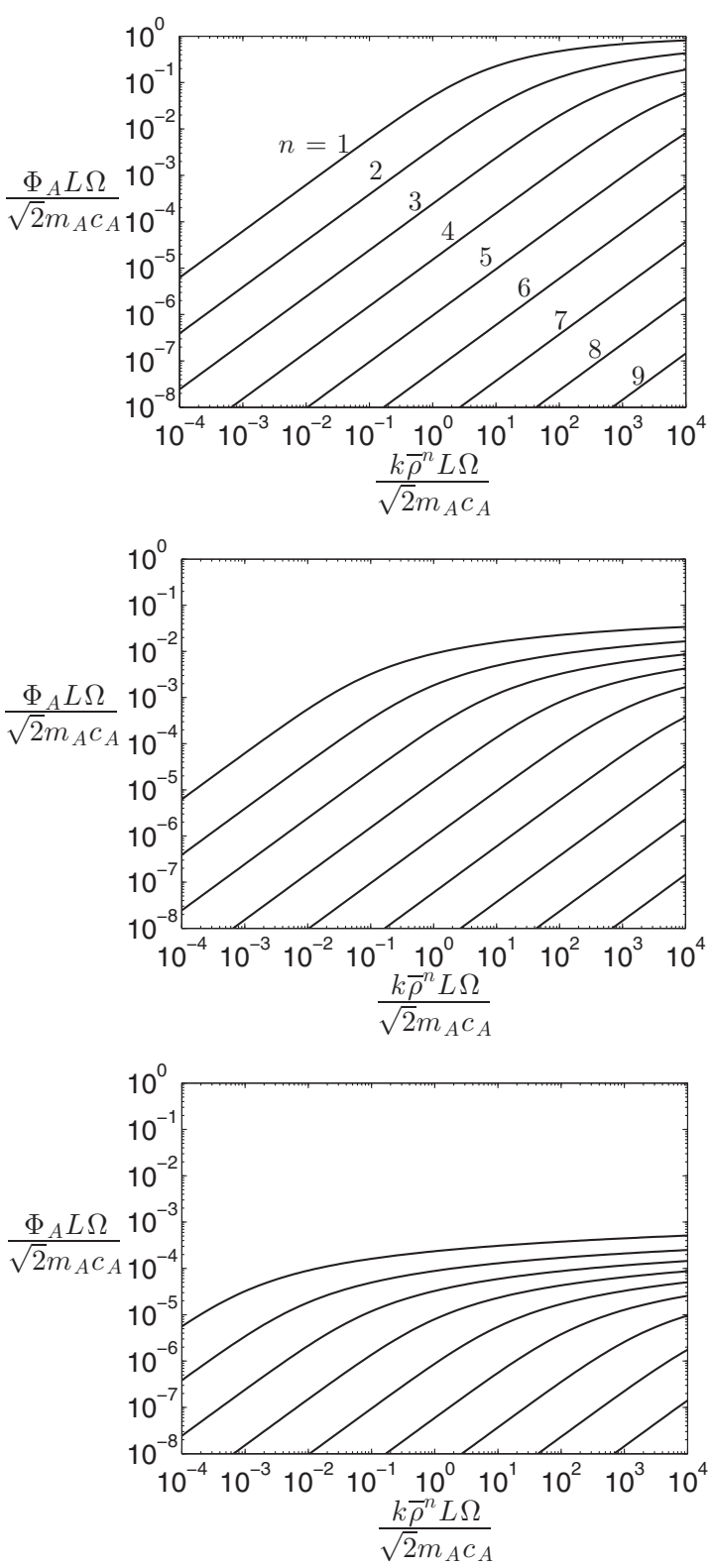

FIG. 1. The analytical solution to the mass flux, as predicted by Eq. (C5) for $c_{B} / c_{A}=1$ (top), $c_{B} / c_{A}=10$ (middle), and $c_{B} / c_{A}=10^{2}$ (bottom).

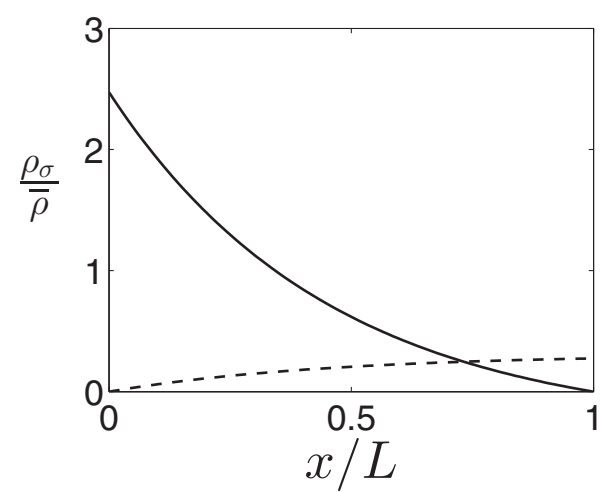

FIG. 2. The analytical solution to the density profiles of component $A$ (solid line) and component $B$ (dashed line) for $c_{B} / c_{A}=3$ and $\kappa L=2 \log \left(c_{B} / c_{A}\right) \approx 2.2$, where $\kappa$ is defined in Eq. (C3d). role. However, when advection is concerned, one needs to be able to tune the diffusivity and the viscosity separately, which can be done, for example, using methods, that involve multiple relaxation times per component; see, e.g., Refs. [13,29].

By inserting Eq. (18) into Eq. (14), the surface reaction bounce-back method specializes into

$$
\begin{aligned}
& \left\{\begin{array}{l}
f_{A, \bar{\alpha}}^{+}=f_{A, \alpha}^{-}+6\left(\frac{\Delta t}{\Delta x}\right)^{2} w_{\alpha} k \rho_{B}^{n}\left[1+\frac{\Delta x n k \rho_{B}^{n-1}}{2 D_{B}}\right]^{-1} \\
f_{B, \bar{\alpha}}^{+}=f_{B, \alpha}^{-}-6\left(\frac{\Delta t}{\Delta x}\right)^{2} w_{\alpha} k \rho_{B}^{n}\left[1+\frac{\Delta x n k \rho_{B}^{n-1}}{2 D_{B}}\right]^{-1}
\end{array}\right\} \text { for } x=0, \\
& \left\{\begin{array}{l}
f_{A, \bar{\alpha}}^{+}=f_{A, \alpha}^{-}-6\left(\frac{\Delta t}{\Delta x}\right)^{2} w_{\alpha} k \rho_{A}^{n}\left[1+\frac{\Delta x n k \rho_{A}^{n-1}}{2 D_{A}}\right]^{-1} \\
f_{B, \bar{\alpha}}^{+}=f_{B, \alpha}^{-}+6\left(\frac{\Delta t}{\Delta x}\right)^{2} w_{\alpha} k \rho_{A}^{n}\left[1+\frac{\Delta x n k \rho_{A}^{n-1}}{2 D_{A}}\right]^{-1}
\end{array}\right\} \text { for } x=L,
\end{aligned}
$$

where $D_{\sigma}$ [Eq. (17)] and $\rho_{\sigma}$ are evaluated at the nearest lattice site to the wall, which is at $x=\Delta x / 2$ and $x=L-\Delta x / 2$ for the reactions at $x=0$ and $x=L$, respectively. Simulations are conducted of Eqs. (4), (19), and (20). The initial conditions for the simulations are flat density profiles: $\rho_{A}(x)=\rho_{B}(x)=\bar{\rho} / 2$. While integrating in time the reaction mass flux $\Phi_{A}$ is monitored. Time integration is stopped when a steady state is reached; i.e., when the variation of $\Phi_{A}$ per time step becomes smaller than $10^{-12} \Phi_{A}$. The purpose of these simulations is to study the error that is introduced by the surface reaction bounce-back method [Eq. (14)]. In the present test case $R=k \rho_{A}^{n}$, and the error estimate [Eq. (15)] becomes

$$
\epsilon=\left[\frac{n}{\rho_{A}} \frac{\partial^{2} \rho_{A}}{\partial x^{2}}+\frac{n[n-1]}{\rho_{A}^{2}}\left[\frac{\partial \rho_{A}}{\partial x}\right]^{2}\right] \frac{\Delta x^{2}}{8} .
$$

As previously mentioned, the error estimate is due to reaction curvature and density curvature. To verify these influences separately, two sets of simulations are conducted, with varying diffusivities, reaction rate constants, reaction rate orders, and sound speeds. The parameters of the simulations are given in the caption of Fig. 3.

The first set of simulations concerns first-order reactions ( $n=1)$, and nonequal sound speeds $\left(c_{B} / c_{A}=2,3,4\right.$, and 5$)$. In this case the reaction curvature is zero while the density curvature is nonzero and the error estimate is given by

$$
\epsilon=\frac{\kappa^{2} \Delta x^{2}}{8}
$$

where $\kappa$ is given by Eq. (C3c). Figure 3(a) shows the computed error, which is the difference between the simulated mass flux and Eq. (C5) as a function of $(\kappa \Delta x)^{2}$. The figure shows that the simulation data collapse onto a linear relation between the error and $(\kappa \Delta x)^{2}$, which is in agreement with Eq. (22).

The second set of simulations concerns equal sound speeds $\left(c_{A} / c_{B}=1\right)$, and higher-order reaction rates $(n=4,9,16$, and 25), which implies a zero density curvature and a nonzero reaction curvature, such that the error estimate becomes

$$
\epsilon=\frac{n[n-1] \tilde{\kappa}^{2} \Delta x^{2}}{8},
$$

where $\tilde{\kappa}$ is given by Eq. (C4c). Figure 3(b) also shows a collapse of the simulation data onto a linear relationship between the error and $n(n-1)(\tilde{\kappa} \Delta x)^{2}$, in agreement with Eq. (23). 



FIG. 3. The relative difference between the mass flux predicted by the LB simulation and by Eq. (C5). (top) Effect of density curvature. Parameters: $L / \Delta x=4, c_{B} \Delta t / \Delta x=1 / \sqrt{3}$, $n=1, \quad\left[m_{A}+m_{B}\right] / \bar{\rho} \Delta t \Omega \sqrt{c_{A}^{2}+c_{B}^{2}}=\left\{\begin{array}{lll}0.2, & 0.53,1.4,3.8,10\end{array}\right.$, $k \Delta t / \Delta x=\{0.010,0.032,0.1,0.32,1.0\}$, and $c_{B} / c_{A}=2(\square), 3(\nabla)$, $4(\triangle), 5(\diamond)$. The dashed line represents $y \sim x$. (bottom) Effect of reaction curvature. Parameters: $L / \Delta x=4, c_{B} \Delta t / \Delta x=1 / \sqrt{3}$, $c_{A} / c_{B}=1,\left[m_{A}+m_{B}\right] / \bar{\rho} \Delta t \Omega \sqrt{c_{A}^{2}+c_{B}^{2}}=\{0.2,0.53,1.4,3.8,10\}$, $k \Delta t / \Delta x=\{0.010,0.032,0.1,0.32,1.0\}$, and $n=4(\square), 9(\nabla), 16$ $(\triangle), 25(\diamond)$. The dashed line represents $y \sim x$.

These simulation results confirm that the error in the present surface reaction bounce-back method [Eq. (14)] is due to reaction curvature and density curvature as predicted by Eq. (15). This also implies that the error scales with the lattice spacing squared, as predicted by Eq. (15); i.e., the method is second-order accurate. It is noted that the difference between the simulated mass flux and Eq. (C5) is of machine precision when there is neither reaction curvature $(n=1)$ nor density curvature $\left(c_{A} / c_{B}=1\right)$.

\section{CONCLUSIONS}

A method [Eq. (14)] is presented to implement surface reaction boundary conditions in a multicomponent lattice Boltzmann framework. By applying the method to a test case and comparing the results to the corresponding analytical solution, the method is shown to be second-order accurate; i.e., the error introduced by the method scales with the square of the lattice spacing.

\section{APPENDIX A: VELOCITY VECTORS AND DISTRIBUTION WEIGHTS}

TABLE I. Lattice Boltzmann velocity vectors $\boldsymbol{v}_{\alpha}$ and distribution weights $w_{\alpha}$ for three commonly used velocity sets in $D=1,2$, and 3 dimensions, consisting of $Q=3,9$, and 19 velocities, which are referred to as D1Q3, D2Q9, and D3Q19, respectively.

\begin{tabular}{|c|c|c|c|c|c|}
\hline $\begin{array}{l}\text { D1Q3 } \\
\boldsymbol{v}_{\alpha} \frac{\Delta t}{\Delta x} \\
\end{array}$ & $\begin{array}{c}\mathrm{D} 1 \mathrm{Q} 3 \\
w_{\alpha}\end{array}$ & $\begin{array}{l}\text { D2Q9 } \\
\boldsymbol{v}_{\alpha} \frac{\Delta t}{\Delta x}\end{array}$ & $\begin{array}{c}\text { D2Q9 } \\
w_{\alpha} \\
\end{array}$ & $\begin{array}{c}\text { D3Q19 } \\
\boldsymbol{v}_{\alpha} \frac{\Delta t}{\Delta x}\end{array}$ & $\begin{array}{c}\text { D3Q19 } \\
w_{\alpha} \\
\end{array}$ \\
\hline 0 & $\frac{2}{3}$ & $(0,0)$ & $\frac{4}{9}$ & $(0,0,0)$ & $\frac{1}{3}$ \\
\hline-1 & $\frac{1}{6}$ & $(-1,0)$ & $\frac{1}{9}$ & $(-1,0,0)$ & $\frac{1}{18}$ \\
\hline \multirow[t]{17}{*}{1} & $\frac{1}{6}$ & $(1,0)$ & $\frac{1}{9}$ & $(1,0,0)$ & $\frac{1}{18}$ \\
\hline & & $(0,-1)$ & $\frac{1}{9}$ & $(0,-1,0)$ & $\frac{1}{18}$ \\
\hline & & $(0,1)$ & $\frac{1}{9}$ & $(0,1,0)$ & $\frac{1}{18}$ \\
\hline & & $(-1,-1)$ & $\frac{1}{36}$ & $(0,0,-1)$ & $\frac{1}{18}$ \\
\hline & & $(1,-1)$ & $\frac{1}{36}$ & $(0,0,1)$ & $\frac{1}{18}$ \\
\hline & & $(-1,1)$ & $\frac{1}{36}$ & $(-1,-1,0)$ & $\frac{1}{36}$ \\
\hline & & $(1,1)$ & $\frac{1}{36}$ & $(1,-1,0)$ & $\frac{1}{36}$ \\
\hline & & & & $(-1,1,0)$ & $\frac{1}{36}$ \\
\hline & & & & $(1,1,0)$ & $\frac{1}{36}$ \\
\hline & & & & $(-1,0,-1)$ & $\frac{1}{36}$ \\
\hline & & & & $(1,0,-1)$ & $\frac{1}{36}$ \\
\hline & & & & $(-1,0,1)$ & $\frac{1}{36}$ \\
\hline & & & & $(1,0,1)$ & $\frac{1}{36}$ \\
\hline & & & & $(0,-1,-1)$ & $\frac{1}{36}$ \\
\hline & & & & $(0,1,-1)$ & $\frac{1}{36}$ \\
\hline & & & & $(0,-1,1)$ & $\frac{1}{36}$ \\
\hline & & & & $(0,1,1)$ & $\frac{1}{36}$ \\
\hline
\end{tabular}

\section{APPENDIX B: BINARY DIFFUSION}

Here a derivation is presented for the diffusivity in a binary ideal gas mixture consisting of molecules of mass $m_{\sigma}$, where $\sigma=1,2$. During binary diffusion, the molecules of both types move on average with different component velocities $u_{\sigma}$. Consider a coordinate system that moves with the mass averaged velocity $u=\sum_{\sigma} u_{\sigma} \rho_{\sigma} / \sum_{\sigma} \rho_{\sigma}$, such that in this coordinate system $u=0$. Here $\rho_{\sigma}$ is the component mass density and $\rho=\sum_{\sigma} \rho_{\sigma}$ is the total mass density. Due to friction, a single molecule of type $\sigma$ experiences an average force $F_{\sigma}$. This force is written as the product of the average momentum exchange $\Delta \pi_{\sigma}$ due to a single collision between $m_{1}$ and $m_{2}$ and the collision frequency $\omega_{\sigma}$ :

$$
F_{\sigma}=\Delta \pi_{\sigma} \omega_{\sigma}
$$

For the sake of simplicity a 1D collision model is used:

$$
\begin{aligned}
\Delta \pi_{1} & =\frac{2 m_{1} m_{2}\left[u_{2}-u_{1}\right]}{m_{1}+m_{2}}, \\
\Delta \pi_{2} & =\frac{2 m_{1} m_{2}\left[u_{1}-u_{2}\right]}{m_{1}+m_{2}},
\end{aligned}
$$


and the collision frequency is written as

$$
\begin{aligned}
& \omega_{1}=\frac{1}{2} n_{2} \Omega \sqrt{c_{1}^{2}+c_{2}^{2}}, \\
& \omega_{2}=\frac{1}{2} n_{1} \Omega \sqrt{c_{1}^{2}+c_{2}^{2}},
\end{aligned}
$$

where $c_{\sigma}=\sqrt{k_{B} T / m_{\sigma}}$ is the sound speed of component $\sigma, k_{B}$ is the Boltzmann constant, $T$ is the temperature, $n_{\sigma}=\rho_{\sigma} / m_{\sigma}$ is the number density of component $\sigma$, and $\Omega$ is a collisional cross section. Combining Eqs. (B1)-(B3) gives

$$
\begin{aligned}
& F_{1}=\zeta n_{2}\left[u_{2}-u_{1}\right], \\
& F_{2}=\zeta n_{1}\left[u_{1}-u_{2}\right],
\end{aligned}
$$

where

$$
\zeta=\frac{\Omega \sqrt{c_{1}^{2}+c_{2}^{2}} m_{1} m_{2}}{m_{1}+m_{2}},
$$

is a constant. Note that Eq. (B4) satisfies the overall force balance $n_{1} F_{1}+n_{2} F_{2}=0$. Over small enough distances, the force $F_{\sigma}$ is a constant, which corresponds to a potential energy of $-x F_{\sigma}$. The probability $n_{\sigma}$ for finding a molecule at location $x$ is therefore given by the Boltzmann distribution:

$$
\begin{aligned}
& n_{1}=n_{1,0} \exp \frac{\zeta n_{2}\left[u_{2}-u_{1}\right] x}{k_{B} T}, \\
& n_{2}=n_{2,0} \exp \frac{\zeta n_{1}\left[u_{1}-u_{2}\right] x}{k_{B} T},
\end{aligned}
$$

where $n_{\sigma, 0}$ are constants. Differentiating Eq. (B5) w.r.t. $x$ gives

$$
\begin{aligned}
\frac{\partial n_{1}}{\partial x} & =\frac{\zeta\left[u_{2}-u_{1}\right] n_{1} n_{2}}{k_{B} T}, \\
\frac{\partial n_{2}}{\partial x} & =\frac{\zeta\left[u_{1}-u_{2}\right] n_{1} n_{2}}{k_{B} T} .
\end{aligned}
$$

Note that according to Eq. (B6) $\frac{\partial n_{1}}{\partial x}=-\frac{\partial n_{2}}{\partial x}$, which means that in this analysis, there is no gradient in the pressure $p=$ $k_{B} T\left[n_{1}+n_{2}\right]$. Combining Eq. (B6) with the fact that in the comoving reference frame there is no net mass flux: $n_{1} m_{1} u_{1}+$ $n_{2} m_{2} u_{2}=0$, gives

$$
\begin{aligned}
& n_{1} u_{1}=-\frac{m_{2} k_{B} T}{\rho \zeta} \frac{\partial n_{1}}{\partial x}, \\
& n_{2} u_{2}=-\frac{m_{1} k_{B} T}{\rho \zeta} \frac{\partial n_{2}}{\partial x} .
\end{aligned}
$$

By combining Eqs. (B4c) and (B7) the component mass flux $\Phi_{\sigma}$ is obtained:

$$
\Phi_{\sigma}=\rho_{\sigma} u_{\sigma}=-D_{\sigma} \frac{\partial \rho_{\sigma}}{\partial x}
$$

where the component mass diffusivity is given by

$$
D_{\sigma}=\frac{c_{\sigma}^{2}\left[m_{1}+m_{2}\right]}{\rho \Omega \sqrt{c_{1}^{2}+c_{2}^{2}}} .
$$

\section{APPENDIX C: ANALYTICAL SOLUTION TO THE TEST CASE}

Here the "analytical" solution is presented, which in fact is obtained by iteration, for the reaction mass flux in the test case, which is described in Sec. V. Since the wall molecules do not participate in the chemical reaction, the total mass balance is given by

$$
\Phi_{A}+\Phi_{B}=0 .
$$

Furthermore the mass fluxes are expressed in terms of the reaction rates [Eq. (18a)]:

$$
\begin{array}{ll}
\Phi_{A}=k \rho_{B}^{n} & \text { for } x=0, \\
\Phi_{B}=-k \rho_{A}^{n} & \text { for } x=L,
\end{array}
$$

where the minus sign reflects that at $x=L$ the wall normal points in the negative $x$-direction. Since the system is steady and $1 \mathrm{D}$ and since there is no mass averaged velocity $u=0$, the component mass mass balance [Eq. (5)] is reduced to

$$
\Phi_{\sigma}=-D_{\sigma} \frac{\partial \rho_{\sigma}}{\partial x}
$$

The density profiles $\rho_{A}(x)$ and $\rho_{B}(x)$ are governed by Eqs. (16), (17), (18b), (C1), and (C2). Depending on the sound speed ratio $c_{A} / c_{B}$, there are two types of solutions. When $c_{A} / c_{B} \neq 1$, the profiles are exponential functions:

$$
\rho_{A}=\frac{p}{c_{A}^{2}-c_{B}^{2}}-\frac{c_{B}^{2} \bar{\rho} L \kappa \exp \{-\kappa x\}}{\left[c_{A}^{2}-c_{B}^{2}\right][1-\exp \{-\kappa L\}]}
$$

and

$$
\rho_{B}=\frac{p}{c_{B}^{2}-c_{A}^{2}}-\frac{c_{A}^{2} \bar{\rho} L \kappa \exp \{-\kappa x\}}{\left[c_{B}^{2}-c_{A}^{2}\right][1-\exp \{-\kappa L\}]} .
$$

Here $p$ is the $x$-independent fluid pressure:

$$
p=\rho \sum_{\sigma} c_{\sigma}^{2}
$$

and $\kappa$ is given by

$$
\kappa=\frac{\Phi_{A}\left[\frac{1}{c_{A}^{2}}-\frac{1}{c_{B}^{2}}\right] \Omega \sqrt{c_{A}^{2}+c_{B}^{2}}}{m_{A}+m_{B}} .
$$

For the special case $c_{A} / c_{B}=1$, the profiles are linear functions:

$$
\rho_{A}=\bar{\rho}\left\{\frac{1}{2}-\tilde{\kappa} L\left[\frac{x}{L}-\frac{1}{2}\right]\right\}
$$

and

$$
\rho_{B}=\bar{\rho}\left\{\frac{1}{2}+\tilde{\kappa} L\left[\frac{x}{L}-\frac{1}{2}\right]\right\},
$$

where $\tilde{\kappa}$ is given by

$$
\tilde{\kappa}=\frac{\Phi_{A} \Omega}{\sqrt{2} c_{A} m_{A}} .
$$

Combining Eqs. (18a) and (C3) gives the following equation for the mass flux when $c_{A} / c_{B} \neq 1$ :

$$
\zeta=\log \left[\frac{2\left[\frac{\beta \zeta^{1-n}}{k \bar{\rho}^{n}}\right]^{\frac{1}{n}}+\frac{c_{B}^{2}}{c_{B}^{2}-c_{A}^{2}}}{2\left[\frac{\beta \zeta^{1-n}}{k \bar{\rho}^{n}}\right]^{\frac{1}{n}}+\frac{c_{A}^{2}}{c_{B}^{2}-c_{A}^{2}}}\right],
$$


where $\zeta$ is given by

$$
\zeta=\frac{\Phi_{A}}{\beta},
$$

and $\beta$ is given by

$$
\beta=\frac{m_{A}+m_{B}}{L\left[\frac{1}{c_{A}^{2}}-\frac{1}{c_{B}^{2}}\right] \Omega \sqrt{c_{A}^{2}+c_{B}^{2}}} .
$$

For the special case when $c_{A} / c_{B}=1$ the mass flux is found by combining Eqs. (18a) and (C4):

$$
\tilde{\zeta}=1-2\left[\frac{\tilde{\zeta} \tilde{\beta}}{k \bar{\rho}^{n}}\right]^{\frac{1}{n}}
$$

where $\tilde{\zeta}$ is given by

$$
\tilde{\zeta}=\frac{\Phi_{A}}{\tilde{\beta}}
$$

and $\tilde{\beta}$ is given by

$$
\tilde{\beta}=\frac{\sqrt{2} c_{A} m_{A}}{L \Omega} .
$$

The solution $\Phi_{A}$ to Eq. (C5) is obtained by iteration until the variation per iteration becomes smaller than $10^{-12} \Phi_{A}$. Figure 1 shows this mass flux as a function of the independent parameters.
[1] R. Benzi, S. Succi, and M. Vergassola, Phys. Rep. 222, 145 (1992).

[2] S. P. Dawson, S. Chen, and G. D. Doolen, J. Chem. Phys. 98, 1514 (1993).

[3] S. Chen and G. D. Doolen, Ann. Rev. Fluid Mech. 30, 329 (1998).

[4] D. A. Wolf-Gladrow, Lattice-Gas Cellular Automata and Lattice Boltzmann Models: An Introduction, Lecture Notes in Mathematics, Vol. 1725 (Springer, Berlin, 2000).

[5] S. Succi, The Lattice Boltzmann Equation: For Fluid Dynamics and Beyond (Oxford University Press, Oxford, 2001).

[6] S. Chen, S. Dawson, G. D. Doolen, D. Janecky, and A. Lawniczak, Comput. Chem. Eng. 19, 617 (1995).

[7] X. He, N. Li, and B. Goldstein, Mol. Simulat. 25, 145 (2000).

[8] Q. Kang, D. Zhang, S. Chen, and X. He, Phys. Rev. E 65, 036318 (2002).

[9] S. Succi, Phys. Rev. Lett. 89, 064502 (2002).

[10] R. Verberg and A. J. C. Ladd, Phys. Rev. E 65, 056311 (2002).

[11] F. Verhaeghe, S. Arnout, B. Blanpain, and P. Wollants, Phys. Rev. E 73, 036316 (2006).

[12] Q. Kang, P. C. Lichtner, and D. Zhang, Water Resour. Res. 43, W12S14 (2007).

[13] S. Arcidiacono, J. Mantzaras, and I. V. Karlin, Phys. Rev. E 78, 046711 (2008).

[14] S. D. C. Walsh and M. O. Saar, Phys. Rev. E 82, 066703 (2010).
[15] M. R. Kamali, S. Sundaresan, H. E. A. Van den Akker, and J. J. J. Gillissen, Chem. Eng. J. 207-208, 587 (2012).

[16] R. Machado, Chem. Eng. Sci. 69, 628 (2012).

[17] T. Zhang, B. Shi, Z. Guo, Z. Chai, and J. Lu, Phys. Rev. E 85, 016701 (2012).

[18] L. Chen, Q. Kang, B. A. Robinson, Y.-L. He, and W.-Q. Tao, Phys. Rev. E 87, 043306 (2013).

[19] C. Huber, B. Shafei, and A. Parmigiani, Geochim. Cosmochim. Ac. 124, 109 (2014).

[20] A. J. C. Ladd and R. Verberg, J. Stat. Phys. 104, 1191 (2001).

[21] Z. Guo, C. Zheng, and B. Shi, Phys. Fluids 14, 2007 (2002).

[22] F. J. Alexander, H. Chen, S. Chen, and G. D. Doolen, Phys. Rev. A 46, 1967 (1992).

[23] M. E. McCracken and J. Abraham, Phys. Rev. E 71, 046704 (2005).

[24] X. Shan and G. D. Doolen, Phys. Rev. E 54, 3614 (1996).

[25] X. He, S. Chen, and G. D. Doolen, J. Comput. Phys. 146, 282 (1998).

[26] S. Ansumali and I. V. Karlin, Phys. Rev. E 66, 026311 (2002).

[27] J. Zhang, Microfluid. Nanofluid. 10, 1 (2011).

[28] T. Cowling and S. Chapman, The Mathematical Theory of Nonuniform Gases (Cambridge University Press, Cambridge, 1960).

[29] Z.-H. Chai and T.-S. Zhao, Acta Mech. Sinica 28, 983 (2012). 